\title{
A Review of the Research on the Relationship Between Color and Donation Behavior
}

\author{
Chunmei Gu \\ School of Business Administration \\ Zhejiang Gongshang University \\ Hangzhou, China
}

\author{
Jiakai Wang \\ School of Business Administration \\ Zhejiang Gongshang University \\ Hangzhou, China
}

\author{
Min Hou \\ School of Business Administration \\ Zhejiang Gongshang University \\ Hangzhou, China
}

\begin{abstract}
In daily life, color affects people all the time. In the field of advertising, image color is more widely used, and the effect of different color advertising images is different. In the field of philanthropy, especially in the research of Internet philanthropy, does the color information of pictures also affect the willingness and behavior of donors? This paper sorts out the related concepts of color and arousal, and uses arousal theory to explain the influence mechanism of picture color information on donation behavior.
\end{abstract}

Keywords-color; donation; arousal

\section{INTRODUCTION}

On the Internet fundraising information platform, information such as pictures and texts displayed by charitable organizations is the main factor affecting donors' willingness to donate. Individual donors make choices for donations based on processing results through processing of donated information. In the process of Internet information dissemination, the content of pictures is more irritating to consumers than text content. In the past, research has been more inclined to study the content displayed in pictures, and to ignore the attributes of pictures (such as color).

In the past, scholars explored the influence of color on individual perception mainly from the following dimensions. In the study of hue dimensions, Goldstein and Costigan believe that the higher the hue value (such as red and yellow) can play an exciting role, causing higher levels of emotional arousal, while hue values of color information (such as blue and purple) will make consumers calm and relaxed. In the study of the saturation dimension, the researchers found that the higher the saturation, the higher the level of emotional arousal [1]. In the study of the brightness dimension, the higher the brightness, the consumer feels relaxed, so when the background color of the advertisement is high, the consumer will positively evaluate the advertisement [2].

In the field of sensory marketing, color influences consumers' emotions, perceptions, and buying behavior [3].
Stroop experiments show that the color of vocabulary interferes with the process of vocabulary recognition, and studies have shown that the color information of product images affects consumers' payment behavior [4]. Since the research results of advertising marketing and sensory marketing show that the color information of product images and background images affect consumers' perceptions and purchasing behavior. Therefore, this study speculates that the color of the image also influence the donor's decision in the field of good cause marketing.

The color information of the public welfare project image can also influence the donor's donation decision. The influencing factors of donation behavior are not only external clues, but the internal psychological factors of individuals also affect the outcome of donations. According to the 2018 workplace report, $80 \%$ of Chinese employees are under different levels of psychological stress, and psychological stress has become one of the psychological characteristics of today's social individuals. Previous studies have also shown that psychological stress affects donors' donation behavior. In some studies, psychological stress has been shown to promote prosocial behavior in young individuals, and in some studies it has been shown to reduce the prosocial behavior of individuals. In addition, psychological stress also significantly affects the individual's emotional arousal state [5] and attention. Therefore, this study speculated that stress plays a moderating role in the process of the influence of picture color information on the donation behavior.

The purpose of this research is to clarify the relationship between color information of pictures and donation behavior by combing the previous literature and deriving related theories. And explore whether there is a difference in the effect of the color information of the picture on the donation behavior under different stress scenarios. 


\section{CONCEPTION}

\section{A. Color}

Color refers to the seven basic colors (red, orange, yellow, green, blue, ochre, purple) in the visible spectrum color and the mixed colors of different colors between them, in a series of color combinations. Alexander and Shansky [6], Labrecque [7] suggest that each color has three attributes, hue, saturation and lightness, of which black and white are special colors, only have a single attribute of brightness without hue and saturation. The hue is the wavelength, which can be divided into cool and warm colors. The brightness is basically the white-black attribute of the color. Saturation is the intensity or vividness of a color [8].

Hue is the most important feature of color and one of the most intuitive features to distinguish colors. In color research, the study of hue is the earliest and most common. The main basis for the division of hue is the wavelength of color light. According to the wavelength, color can be divided into high hue color and low hue color. Among them, red and blue are the main colors. Previous studies have mainly focused on red and blue. In terms of color, these two types of colors mainly affect the individual's cognitive and emotional responses.

The saturation of color is the vividness of color. A lowsaturation color with a high proportion of black and white, look darker. While the high-saturation color with a low proportion of black and white, look vivid.

The lower the brightness of the color, the closer the color looks to black, the weaker the stimulus; the higher the brightness, the closer the color looks to white, the stronger the stimulus, while the black and white only have the brightness attribute, no saturation and hue properties [9].

\section{B. Emotional Arousal}

Emotion is a positive force with perceptual and motivational functions that can play an organizational role in maintaining and guiding individual behavior. This definition is similar to the definition of Arnold and Lazarus. They believe that the generation of emotions is a dual response of individual physical and psychological organizations, based on beneficial or harmful stimuli in the external environment. Different scholars have different understandings of the specific definition of the word emotion.

Arousal is an activation state of the nervous system with high and low poles [10]. In the low arousal state, the individual exhibits the characteristics of relaxation and quietness, while in the high arousal state individuals exhibit characteristics such as concentration. In fact, in the 2011 Brooks study, arousal has been shown to influence individual actions, such as actively seeking help from others and quickly resolving problems encountered in negotiations. Therefore, emotional arousal plays an important role in regulating consciousness, attention, alertness, and information processing.

\section{Attention}

Simon believes that management is decision making, and the key to decision making is how decision makers can effectively allocate their limited attention. Based on Simon's concept of attention, researchers have defined the concept of attention from both the content and process perspectives. In terms of content, attention refers to the stimulus that occupies the consciousness of decision makers in the many stimulus related to decision-making [11]. From the perspective of process, attention allocation refers to decision makers The process of assigning its own limited information processing capabilities to decision-related stimuli, including attention, coding, interpretation, and focus on stimuli.

Marketing scholars pay attention to the introduction of consumer behavior research, pointing out that consumer attention is to focus limited resources on a small amount of information, and prioritize the content of attention at the expense of other information [12].

\section{Psychological Stress}

The concept of stress stems from physiology, and Cannon introduced the concept of stress into the field of sociology. He suggests that stress is a disorder of homeostasis under the influence of external factors, and uses "body balance" to describe the body's response to the stability of the body in the face of environmental changes. Marketing scholars pay attention to the introduction of consumer behavior research, pointing out that consumer attention is to focus limited resources on a small amount of information, and prioritize the content of attention at the expense of other information [13].

According to the transaction model proposed by Lazarus [14], psychological stress refers to the second-stage assessment of stressors after individuals encounter stressors. If they think that measures need to be taken to deal with stressors, they will generate alarms such as nervousness and anxiety. Theory of conservation of resources proposed by Hobfoll [15] suggests that psychological stress can arise when individuals use actual resources to generate actual losses, threats of loss, or failure to invest in resources. Effort and return imbalance model argues that psychological stress is based on a judgment of social reciprocity and social transactions [16]. If the result of the judgment is unbalanced reciprocity, the individual will have strong negative emotions and persistent tension. This process is considered a psychological stress response. The above models have different definitions of psychological stress, but in summary, psychological stress is an unbalanced state. When the external stressors (threats) are unbalanced with their own resources and the resources they can provide, the individual generate psychological stress. For example, when faced with mission impossible, opponents undefeatable or other stressors, the resources that the individual has mastered are insufficient to cope with, and their psychological stress will arise, resulting in tension and anxiety. 


\section{THEORETICAL BACKGROUND}

\section{A. Arousal Theory}

The theory of emotional arousal is proposed by Hagtvedt [17], which mainly describes the relationship between color information such as saturation, emotional arousal, and perceived size. Emotional arousal theory believes that the effect of color saturation on perceived size follows a thirdorder intermediate pathway, in which saturation affects emotional arousal, emotional arousal affects attention, and attention affects individual perception size.

This theory is consistent with observations in nature. Good-looking flowers or toxic worms have bright colors, which give us a high level of emotional arousal and cause us to be alert. In evolution, mature fruits and poisonous insects can cause evoking of our ancestors and attract their attention.

The theory is also consistent with existing research, that the higher the saturation, the higher the level of emotional arousal in the context of advertising, the higher emotional arousal will occur, be alert and focus on the stimulus that trigger this state [18].

\section{B. Biased-competition Model}

The biased competition model believes that when there are more than two objects in the environment, selective attention to some information can increase the activation degree of the corresponding visual cortex and enhance the neural response to the object being noticed. And different objects compete with each other for higher levels of processing, and ultimately the "winner" can control the individual's perception and behavioral response. That is to say, selective attention to a product increases the degree of activation of its corresponding visual cortex, allowing it to acquire more processing, thereby increasing its likelihood of being selected.

In the study of Janiszewski, this theory was used to test the impact of selective attention on consumer product choices [19]. In the experiment, the researchers examined the influence of attention on the choice of the product by manipulating the attention of the subject to the specific product. It has been found that multiple selective attention to a product increases the likelihood that the product will be selected, and that the product that receives the attention is more likely to be selected.

\section{Resource Constraint Theory}

The resource limitation theory was proposed by Kahneman, which argues that the use of psychological resources is required to complete each task. Several tasks can be used to share mental resources, but the total amount of human psychological resources is limited. These processes produce a certain amount of output, and when a person performs several tasks, the amount of resources is allocated to the operations of these tasks based on the change in the quality of a certain number of resources and outputs. Kahneman regards attention as a psychological resource and believes that the total amount of human psychological resources is limited.

\section{INFLUENCE MECHANISM}

\section{A. Donation}

Scholars believe that social identity is the main influencing factor of donation behavior [20], and the Bystander effect can explain this very well, that is, when the donor thinks others will help, actively classify themself as a "bystander" and reduce the willingness to provide help. The environmental brightness of the consumer [21] and the image information used in the project communication process all affect the individual's donation behavior.

The psychological factors of the donor also affect the donation behavior. When the donor's altruistic motivation is high, their willingness to donate is stronger. Empathy theory indicates that the amount of donation is mainly influenced by self-motivation, and it will be reduced under psychological stress [22]. The anxiety and depression caused by social exclusion will also weaken the mediating effect of empathy, thereby reducing individuals such as charity. These studies show that individual psychological state and psychological stress levels influence the donation behavior.

In addition, personal characteristics such as personal education level, income level, age, and religious beliefs [23] all affect individual donation behavior. However, under different cultural backgrounds, the results of these impact mechanisms are not consistent, and the actual situation in each region is widely confirmed.

\section{B. Color and Arousal}

Hue affects the level of emotional arousal of consumers. When people face different colors of products, they tend to have higher hue of warm colors, mainly because warm colors can evoke positive emotions, and cold colors with lower hue can evoke negative emotions.

In the hue circle, red is a high hue color and blue is a low hue color. Red can trigger higher levels of emotional arousal for consumers, attracting individual attention, and red also motivates individuals to compete [24]. In the online auction environment, consumers' bids in the high hue background are significantly higher than those in the low hue background group. This means that higher hue color may generate higher levels of emotional arousal in individuals, leading to impulsive buying behavior and aggressive behavior [25]. Jacobs [26], Labrecque and Milne [27] suggest that low hue color can bring calm and relaxation to the individual. In the blue background, the consumer's mood is more calm, rational and rational consumption. Therefore, the hue positively affects the emotional arousal. The higher the hue value of the picture color, the higher the level of emotional arousal.

Saturation significantly affects emotional arousal. A more saturated color causes a greater degree of euphoria and is associated with a high arousal, so the color of the different saturations in the advertising is used to excite the individual. There is a difference in the degree of arousal. Highsaturation colors are more attractive to consumers, and consumers are more willing to consume. In the online environment, the effect of saturation on emotional arousal 
will be further enhanced. Therefore, when the purpose of the advertisement is to make the consumer feel relaxed and pleasant, the color with lower saturation is often selected as the background color of the advertisement. So, the saturation positively affects the individual's emotional arousal and the higher the saturation value of the picture color, the higher the level of emotional arousal.

Lightness, like hue and saturation, significantly affects individual emotional arousal. In the use of advertising, the background of high-definition makes the consumer's mood more relaxed than the low-light background, leading to more positive product evaluation by consumers. In another study, it was found that environmental brightness significantly affects consumers' emotional arousal levels, consumers' willingness to purchase increases in high-lightness environments, and consumers' willingness to touch increases significantly when exhibiting products in brighter environments [28]. Therefore, brightness affects the individual's emotional arousal.

\section{Emotional Arousal and Donation}

Emotional arousal can affect an individual's prosocial behavior. Holloway, Tucker, and Hornstein distinguish that the positive emotions allow individuals to pay more attention to their goodwill and mutual help behavior, thereby promoting the growth of helping behavior. When individuals are in a negative mood, people's acceptability of behaviors and events that violate ethics is significantly reduced [29]. Conversely, when an individual is in a positive emotional arousal, the subject is more inclined to engage in prosocial behavior such as donation.

By analyzing the state data of the US presidential elections, the Jasper research team found that high temperatures can trigger higher arousal states, and the temperature is positively correlated with voting support rates [30]. Voting is one of prosocial behaviors. This is consistent with the theory of excitement transmission, in which the level of emotional arousal generated by altitude can be transferred to other activities and play a similar role in these activities.

Similarly, a study in 2017 also showed that the level of arousal was positively correlated with prosocial behavior, and the researchers assessed the relationship between pupil dilation and help to others from 18 months to 5.5 years [31]. The level of physiological arousal produced by pupil dilation was found to positively affect the help behavior of these children. When children observe an individual in need of help, whether they help themselves or a third-party help, their level of arousal is consistent and significantly different from those help seeker. This indicates that the level of physical arousal of the prosocial behavior and the pro-social behavioral individual is consistent and significantly higher than that of the individual who did not witness prosocial behavior. When external stimuli become sources of stress, the prosocial behavior of the individual is also affected. And stimuli such as psychological stress affect the individual's emotional arousal state, and emotional arousal plays a mediating role in this process [32]. These studies have shown that emotional arousal affects the individual's internal altruism, and the altruism influence donation behavior of donors.

\section{The Moderating Effect of Stress}

Psychological stress reduces attention to irrelevant stimuli [33] and increases memory of stress-related stimuli, suggesting information processing bias during stress periods [34]. Experience stress may lead to more accurate processing of stressor-related information, but less accurate processing of other information [35]. According to the theory of resource limitation, individual attention as a Cognitive resources are generally limited. This also indicates that the higher the individual's psychological stress, the more attention is assigned to the task processing related to the stress source, and the total remaining attention resources of the individual becomes less. As the amount of attention is reduced during the donation decision-making process, the attention assigned to the color information of the picture is correspondingly reduced, and the attraction of the project to the donor is reduced.

The biased-competition model indicates that attention affects consumers' decision-making behavior. The more consumers pay attention to a product, the greater the consumer's purchasing probability. Therefore, according to the biased-competition model and the ceiling effect, more attention paid to pictures under low psychological stress, the stronger the donor's willingness to donate; while under high psychological stress, the pictures attract less attention and the donors' willingness to donate is relatively weak.

This is similar to the effect of psychological stress on emotion recognition. In other words, psychological stress reduces the ability of donors to recognize colors of picture. Therefore, psychological stress moderates the effect of picture color information on donation behavior, that is, compared with low psychological stress conditions, under the condition of high psychological stress, the effect of picture color information on donation behavior is weakened.

\section{E. Summary}

Picture color information significantly affects the level of individual arousal [36]. In the field of emotional arousal research, individuals in high arousal state have the characteristics of concentration. Current research indicates that emotional arousal levels positively affect prosocial behavior, and that prosociality is significantly increased in individuals with high emotional arousal levels. Therefore, under the Internet donation scenario, the color information of the pictures displayed by the public welfare project significantly affects the emotional arousal level of the donor, which in turn affects the donor's willingness to donate and donation behavior.

This conclusion is consistent with the theory of emotional arousal. According to the theory of emotional arousal, the higher the saturation is, the stronger the stimulation of color will be and the higher the level of individual arousal will be. Under the condition of high arousal, the individual is more focused, and the product 
attracts more consumers' attention. According to the attention-biased competition model, selective attention increase the activation degree of the corresponding visual cortex, enhance the neural response to the object being noticed, and obtain more processing, thereby increasing the possibility of being selected. Therefore, the more likely the consumer is to purchase the product [37]. Based on the same theoretical background, high-brightness colors can produce higher levels of arousal than low-brightness colors, attracting consumers' attention and increasing consumer product evaluation and purchase intention. Attention is one of the important factors of empathic response, which affects individual's donation behavior and intention [38].Therefore, under the conditions of Internet donation, the more intense the color information of the project picture is, the more attractive it is to attract the attention of the donor, and the more likely the donor will donate for this project.

Psychological stress plays a role in regulating this process. The psychological stress level of the donor affects the total amount of attention, which in turn affects the process of the influence of the color information of the picture on the attention, and finally affects the donor's willingness to donate and the donation behavior. That is, under the condition of high psychological stress, the influence of picture color information on donation behavior is weaker. Under the condition of low psychological stress, the influence of picture color information on donation behavior is more significant.

\section{CONCLUSION}

Research in the field of color marketing and sensory marketing shows that color information affects consumers' information processing capabilities, emotions and cognition. Product images of different colors have different appeals to consumers, and the brightness of the consumer environment and the saturation of the product packaging affect the attention of consumers. These studies have shown that image color information affects consumer behavior.

In the field of good cause marketing, according to the theory of emotional arousal, the color information of the pictures of the donation project can also affect the donation willingness and donation behavior. The color information arouses the state by affecting the emotion of the consumer, attracts the attention of the fundraiser, and finally affecting their willingness to donate and donation behavior. This conclusion is also consistent with the existing findings that individuals have significantly increased prosociality under conditions of higher levels of emotional arousal.

However, at this stage, there is no corresponding empirical research to prove the impact of image color information on donation behavior. This is also the next research direction of this study, trying to verify the influence of color on donation behavior through experimental methods. Future research can mainly expand the influence of color on donation behavior from the following two aspects:

First, conduct empirical research, collect data from Internet donation platforms at home and abroad, and conduct empirical research.
Second, look for the influence mechanism of color information on donation behavior from other angles. Emotional arousal theory is just one of them. Colors also influence the individual's cognitive process and information processing process. This phenomenon can be explained from another angle.

\section{REFERENCES}

[1] Booth, Rob, and D. Sharma. "Stress reduces attention to irrelevant information: Evidence from the Stroop task." Motivation \& Emotion 33.4(2009):412-418.

[2] Gorn, Gerald J., A. Chattopadhyay, and Y. D. W. Dahl. "Effects of color as an executional cue in advertising: Theyl"re in the Shade." Management Science 43.10(1997):1387-1400.

[3] Elliot, Andrew J., and M. A. Maier. "Color psychology: effects of perceiving color on psychological functioning in humans." Annual Review of Psychology 65.1(2014):95-120.

[4] Cheema, Bagchi Amar. "The Effect of Red Background Color on Willingness-to-Pay: The Moderating Role of Selling Mechanism." Journal of Consumer Research 39.5(2013):947-960.

[5] Lazarus, Rs, S. Folkman, and R. Lazarus. "Psychological stress and the coping process." Science 156(1966)

[6] Alexander, Kenneth R., and M. S. Shansky. "Influence of hue, value, and chroma on the perceived heaviness of colors." Attention Perception \& Psychophysics 19.1(1976):72-74.

[7] Labrecque, Lauren I., V. M. Patrick, and G. R. Milne. "The marketers' prismatic palette: A review of color research and future directions." Psychology \& Marketing 30.2(2013):187-202.

[8] Fairchild, Mark D. . "Color appearance models, 2nd Edition." (2005).

[9] Sunaga, Tsutomu, J. Park, and C. Spence. "Effects of lightnesslocation congruency on consumers' purchase decision-making." Psychology \& Marketing 33.11(2016):934-950.

[10] Hong Li, and Feifei Liu. "Review and prospect of marketing information sharing based on emotional perspective." Foreign Economies and Management 9(2018):143-152.

[11] Pieters, Rik, M. Wedel, and R. Batra. "The stopping power of advertising: Measures and effects of visual complexity." Journal of Marketing 74.5(2010):48-60.

[12] Venkatraman, Vinod, et al. "Predicting advertising success beyond traditional measures: New insights from neurophysiological methods and market response modeling." Social Science Electronic Publishing 52.4(2014)

[13] Hobfoll, Stevan E.. "Conservation of resources: A new attempt at conceptualizing stress." American Psychologist 44.3(1989):513-524.

[14] Lazarus, Rs, S. Folkman, and R. Lazarus . "Psychological stress and the coping process." Science 156(1966): 426-453.

[15] Siegrist, Michael, R. Leins-Hess, C. Keller. "Which front-of-pack nutrition label is the most efficient one? The results of an eye-tracker study." Food Quality and Preference 39(2015):183-190.

[16] Lee, Yu Kang, C. T. Chang. "WHO GIVES WHAT TO CHARITY? CHARACTERISTICS AFFECTING DONATION BEHAVIOR." Social Behavior \& Personality: an international journal 66.35(2007):1173-1180.

[17] Bekkers, René, and P. Wiepking. "A literature review of empirical studies of philanthropy." Nonprofit \& Voluntary Sector Quarterly 40.5(2010):924-973.

[18] Hagtvedt, Henrik, S. A. Brasel. "Color saturation increases perceived product size | Journal of Consumer Research | Oxford Academic." Journal of Consumer Research 44.2(2017):ucx039.

[19] Adolphs, R., A. R. Damasio. "The interaction of affect and cognition: A neurobiological perspective." Jp, Forgas Handbook of Affect \& Social Cognition London: Lawrence \& Associates 1 (2000):27-49. 
[20] Janiszewski, Chris, A. Kuo, and N. T. Tavassoli. "The influence of selective attention and inattention to products on subsequent choice." Journal of Consumer Research 39.6(2013):1258-1274.

[21] Ke Zhong, Haizhong Wang, and Chen Yang. " Review and prospect of research on sensory marketing." Foreign Economics and Management 38.5(2016):68-79.

[22] Dickert, Stephan, N. Sagara, and P. Slovic. "Affective motivations to help others: A two-stage model of donation decisions." Journal of Behavioral Decision Making 24.4(2011):361-376.

[23] Twenge, Jean M, et al. "Replenishing connectedness: Reminders of social activity reduce aggression after social exclusion." British Journal of Social Psychology 46.1(2007):205-224.

[24] Elliot, A.J.; Maier, M.A.. "Color-in-context theory". Advances in Experimental Social Psychology 45(2012): 61-125.

[25] Pryke, Sarah R.. "Is red an innate or learned signal of aggression and intimidation?." Animal Behaviour 78.2(2009):393-398.

[26] Jacobs, Keith W., J. F. Suess. "EFFECTS OF FOUR PSYCHOLOGICAL PRIMARY COLORS ON ANXIETY STATE." Perceptual and Motor Skills 41.1(1975):207-210.

[27] Labrecque, Lauren I., V. M. Patrick, G. R. Milne. "The marketers' prismatic palette: A review of color research and future directions." Psychology \& Marketing 30.2(2013):187-202.

[28] Summers, Teresa A, P. R. Hebert. "Shedding some light on store atmospherics: Influence of illumination on consumer behavior." Journal of Business Research 54. 2(2001):145-150.

[29] Schnall, S., et al. "Disgust as embodied moral judgment." Personality and Social Psyhology Bulletin 34.8(2008):1096-1109.

[30] Jasper, Van Assche, et al. "When the heat is on: The effect of temperature on voter behavior in presidential elections." Frontiers in Psychology 8(2017):929.

[31] Hepach, Robert, et al. "The relation between young childrenl"s physiological arousal and their motivation to help others." Neuropsychologia (2017).

[32] Vinkers, Christiaan H., et al. "Time-dependent changes in altruistic punishment following stress." Psychoneuroendocrinology 38.9(2013):1467-1475.

[33] Booth, Rob, and D. Sharma. "Stress reduces attention to irrelevant information: Evidence from the Stroop task." Motivation \& Emotion 33.4(2009):412-418.

[34] Shors, and J. Tracey. "Stressful experience and learning across the lifespan." Annual Review of Psychology 57.1(2006):55-85.

[35] Erbas, Yasemin, et al. "Why I don't always know what I'm feeling: The role of stress in within-person fluctuations in emotion differentiation." Journal of Personality and Social Psychology 115.2(2018):179.

[36] Jing Huang, et al. "Color marketing research: review and prospect." Foreign Economies and Management 40,10(2018): 40-53.

[37] Orquin, Jacob L., and S. Mueller Loose. "Attention and choice: A review on eye movements in decision making." Acta Psychologica 144.1(2013):190-206

[38] Stephan Dickert, Paul Slovic. "Attentional mechanisms in the generation of sympathy." JUDGMENT AND DECISION MAKING 4.4(2010):296-307. 